\title{
Autonomous Underwater Vehicle in Ocean Wastes Cleanup
}

\author{
Aaron Don M. Africa, Gregory James Pe, Bianca Clarisse Tan \\ Department of Electronics and Communications Engineering \\ De La Salle University, Manila \\ 2401 Taft Ave., Malate, Manila 1004, \\ Philippines, aaron.africa@dlsu.edu.ph
}

\begin{abstract}
As the world of technology continues to evolve, innovations and programs are integrated into the societal problems that the world is facing today. As robotics has opened a new chapter in the world of technology, there are so many ways to integrate this innovation and develop it to help solve the social and economic issues occurring, This paper emphasizes on how significant Autonomous Underwater Vehicles are in this world today as it aids in marine monitoring which can help in discoveries and marine protection. This paper also focuses on the function, system, and significant features of an Autonomous Underwater Vehicle. For the function the brief and most basic materials and software to develop the body of the device is studied, the system and process of data acquisition are covered, and the new and probable improvements that can be done shortly are also included in this paper. This study aims to establish the fundamental knowledge, the main functions, and the system of an Autonomous Underwater Vehicle.
\end{abstract}

Key words: Underwater Vehicle, Robotics, Automation, Waste Cleanup

\section{INTRODUCTION}

As digitization and innovations continue to prosper and thrive in this era, it has set an avenue for developing technologies that continue to solve social and economic problems that the world continues to face today. According to [1], Robotics in specific has evolved throughout the years for its convenience, effectiveness, and productivity which then lifts humans from work that may be impossible or burdensome to do. As robotics continues to prosper in so many different fields and dominating markets of different types such as food production, military defense, transportation, health, science, and the like. One field of relevant importance would be the marine environment. Thus, integrating the rapid development of robotics and the marine sector, one technology that begins an era of development and socio-economic change would be Autonomous Underwater Vehicles (AUVs).

From the name itself, Autonomous Underwater Vehicles are Vehicles that can withstand depths of water without direct human control. AUVs have been increasingly popular for these features such that it is affordable, can avoid obstacles and obstructions autonomously, underwater communications, and full function without the use of external energy support.

Although AUVs are efficient, to reach its optimum efficiency certain specifications must be attained. As specified by [2], the important specification requirements an AUV must have would be the depth in which the vehicle can withstand, weight and dimensions, requested autonomy, speed of the vehicle, energy consumption, and navigating system. Also, not only is the specification requirements limited to the vehicle but the plan of which route or obstacles the AUV will encounter should also be considered when creating, designing, programming and utilizing the technology, the impact of coral mounds at multiple frequencies, and velocity measurements of the density when submarines flow.

As technology continues to evolve, AUVs having become increasingly popular and significant for its high-resolution records of the seabeds at extreme depths of water. Although AUVs are efficient enough, there are still many ways to improve its durability in terms of the depths it can withstand, duration, its energy capacity, and response to various signals and obstacles around it.

Based on the study of [3], it is no joke that water pollution continues to affect not just marine ecosystems. A paper as early as 1987 have already been on high alert of what the effects of water pollution are to the sea turtles. This is because most sea turtles travel quite far in the ocean and as such these turtles encounter quite a lot of garbage in the ocean. Another study by [4] outlines the different effects of marine debris on different marine animals. The paper even states that marine debris is viewed as a major threat to the biodiversity of the ecosystem. This is because most of this debris are plastics that can sometimes be ingested by some of the aquatic animals. A study by [5] shows the first records of planktivorous fish from the North Pacific Central Gyre NPCG which ingested different kinds of plastic that accumulated in this area, these fishes who ingested plastic composed of $35 \%$ of the ones observed which have an average of about 2.1 pieces of plastic per fish. Studies such as that of [6] even show that even small amounts of plastic can affect water movement and heat transfer in beaches. These changes can affect different biological lives located at beaches. 


\section{BACKGROUND OF THE STUDY}

As stated by [7], Autonomous Underwater Vehicles otherwise known as AUVs have several significant uses to marine geoscience, commercial, policies, and the sciences. AUVs have continuously become popular at this age for several significant reasons. As stated in a journal by [8], that the earth is composed mainly of bodies of water; thus, some countless waters and seas are difficult to study or penetrate due to its location, geographical form, and depth which is why autonomous underwater vehicles are of significant use since this technology does not depend on direct human control.

Society as developed rapidly, which makes it difficult to ignore that drastic changes in the environment caused by various human activities. As the state of the environment continues to disintegrate discoveries are needed to maintain and improve the life forms, and structures still present in marine life. As stated by [9], traditional monitoring utilizing using an oceanographic vessel to monitor the marine environment is inefficient as it is expensive, time-consuming, and displays very low resolution. Thus, with the use of AUVs, engineers and scientists have found a way to increase the efficiency of both times, space, and cost in monitoring the marine environment.

As stated by [10], AUVs have been of great significance in collecting data in rivers and lakes in Asia, specifically East Asia, South Asia, and Southeast Asia. Because of its autonomous feature, it has made marine monitoring convenient thus helping solve various environmental problems such as the shrinking of lakes, melting of glaciers, and many more. This specific AUV has long-endurance, flexible navigation, lightweight and efficient hydrodynamics which is ideal for obtaining data, seafloor maps, and underwater images.

\section{STATEMENT OF THE PROBLEM}

As stated by [11], The present age can now be considered as the plastic age as the amount of plastic debris continues to rapidly increase over the years. The drastic increase is so massive that it is unidentifiable as to how much debris there is in the bodies of water there are. This is because the ocean is so vast that the debris has reached several remote areas, difficult for humans to monitor.

The rate of plastic continues to increase; however, the measure made to resolve this issue have been so little. More than just the rapid increase of plastic debris, marine fauna is now another issue. In a study conducted by [12], around 4.8 to 12.7 million tons of plastic waste coming from land is thrown into the ocean causing tremendous hazards to wildlife and the health of animals and humans.

\section{SIGNIFICANCE OF THE STUDY}

As stated by [13], there is a dire need to change the ecological disruption occurring in both lands and waters because if no action is done, the hazards do not only reach the marine and terrestrial life and resource but to human health as well. This study has become relevant, as the number of waste and the drastic changes continue to penetrate the waters there are little efforts made to resolve this. This is due to the difficulty to reach areas in the ocean which may either be remote or difficult for humans to monitor. However, with the use of AUVs this problem may be easily resolved. without the use of human control, it may be easier to monitor the state of the ocean and it may also be easier to monitor the changes in the seafloor.

\section{DESCRIPTION OF THE SYSTEM}

The model of the AUV will be of three-part modular structure as patterned from the experimental version by) [14], To enable the model to become lightweight, convenient and conventional the model will be made out of aluminum components and most especially glass fiber composite material for the device to withstand the strong winds and currents. Next, to be able to ensure that the AUVs can function in horizontal and vertical motion three bi-directional thruster of which two will be used for the horizontal planar propulsion while the other will be used for vertical propulsion. These are the essential physical requirements needed for the body of the device which can now be modeled utilizing using CAD software, SOLIDWORKS.

Although creating a model that is lightweight and functional, the program of the system is of equal importance as it can help determine which routes to take, how the device will avoid seafloor ice collisions, which data the device should acquire, and the like. Thus, a vital part of an AUV would be the sensors present. With the sensors present, to be able to arrive or acquire data, the necessary measures must be considered for a field of view that is correct and precise this then aids in efficient and accurate monitoring inspection which is significant in marine geosciences. Thus, the system will collect the input parameters and cycle of checking and rechecking will be done to ensure that the field of view is correct and that the data obtained is also of value. Considering that the system is autonomous, the process will not end until the data acquired is what is needed.

\section{METHODOLOGY}

Apart from selecting the best features for the AUV, it is important to carefully program the field of view of the system. In this way, the AUV is used to its optimum capacity and capability. To further explain the way the system functions a model diagram is provided as seen in Figure 1. 


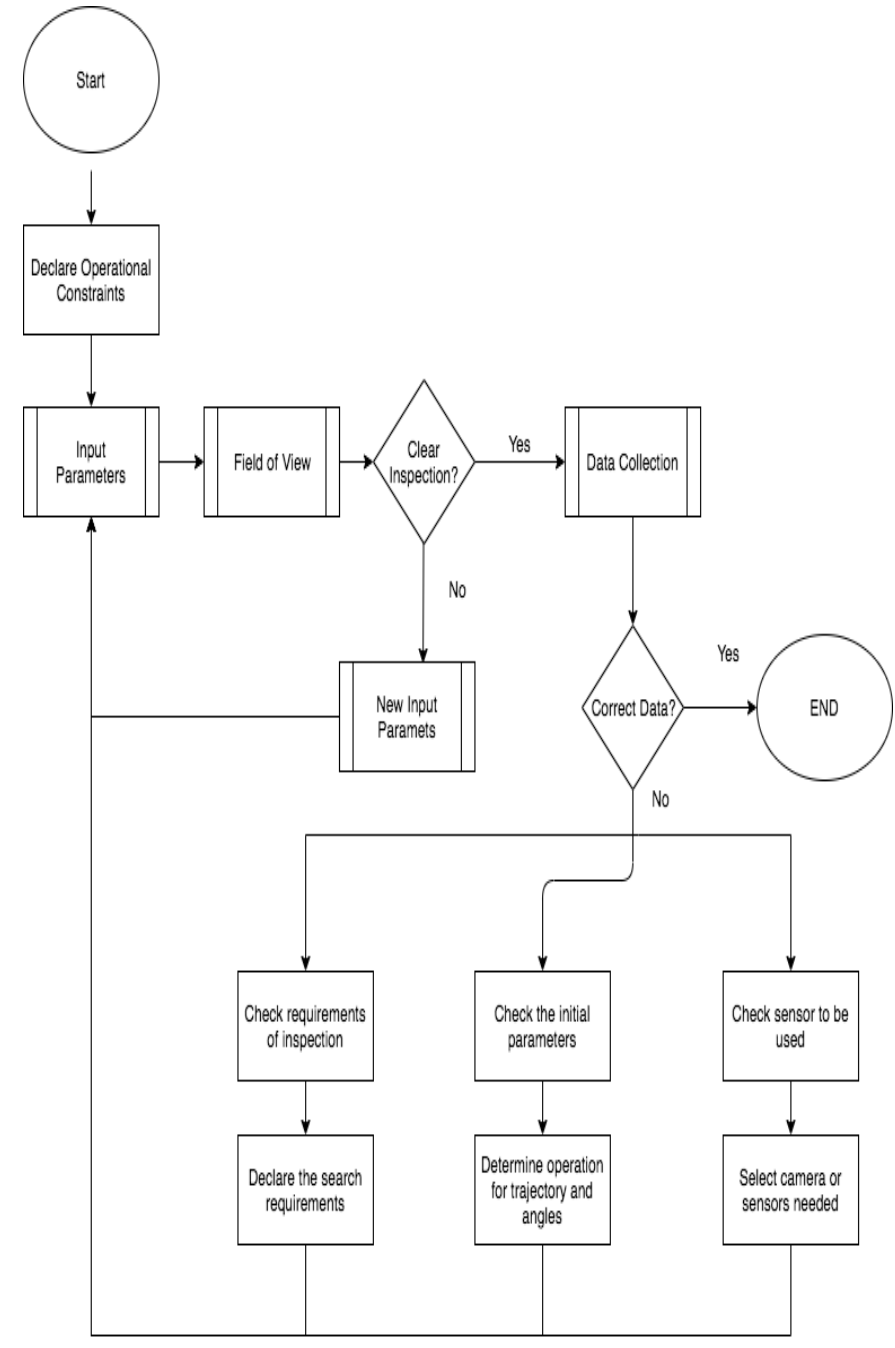

Figure 1: System Flow Chart

First, the system begins by declaring the operational constraints, which means the controls and specifications must be set on the AUV. Second, the AUV will analyze its surroundings and measure these as the input parameters. Third, taking the input parameters, it will then inspect its field of view or what is seen through the vehicle. The next step would be to determine whether the material inspected is correct or not. if the field of view inspection is noted to be incorrect, new input parameters will be needed and this will now replace the initial input parameters. However, if the field of view inspections is noted to be correct it will begin acquiring the data needed. Afterward, the data will be filtered out thus the system will counter check whether the data collected is correct or not. If the system declares that the data is correct, the process will end. If the data collected is wrong, then the system will initialize and check whether the requirements for inspection is met, if the trajectory or the orientation is correct or if the sensors used to meet the requirements are used. Once these parameters are checked the necessary adjustments will be made and the process will repeat back to the input parameters and the cycle continues until the data acquired is accurate enough.

\section{REVIEW OF RELATED LITERATURE}

\subsection{Navigation of AUVs}

The introduction of the inertial navigation back in the early 1930s, as stated by [15], was a feat in early day navigation. To explain, the inertial navigation system is a set of sensors that determines the angle, velocity, altitude, etc. of an object without external references. These systems were prevalent in aircraft and underwater vehicles as an essential tool for navigation. In modern-day AUVs, however, the use of the inertial navigation system presents its own set of inaccuracies. This led to the development of the strap-down and platform inertial navigation system, however, both of these also have their own set of disadvantages. A recent paper by [16] suggests a combination of both the strap down and platform inertial navigation system. Since the strap-down inertial system is weak when it comes to the angular motion of the vehicle, the resulting bench strap-down inertial system eliminates the complication when it comes to the rotation of the vehicle. As such, the researchers in the study concludes of the efficiency of the said bench strap-down inertial system.

As there is a lack of GPS signal, as stated by [17], beneath the waves, researchers have been looking at alternatives in terms of the proper AUV navigation systems. Not only is GPS navigation unreliable, but so is radiofrequency. This is because underwater vehicles can only operate at low bandwidth frequency. Part of this is because of the doppler shift effect which alters the waveform's frequency in underwater communications. Doppler shift is the changing of a frequency due to the source's movement. As such, objects in which the source moves away from receives a lower frequency while that of which the source moves towards to receive a higher frequency when compared to that of the source [18]. This is why underwater communications prove to be a difficult task for these autonomous vehicles.

With this, researchers have sought a better way of navigating an autonomous vehicle under the ocean. The study of [19], describes a new AUV navigation system by using the Unscented Kalman Filter (UKF). To give information on what UKF is, it is the better version of both the Kalman filter, which estimates the state of a linear system, and the Extended Kalman Filter, which is capable of getting the state estimate of a nonlinear but is non-applicable to extremely nonlinear systems as mentioned by the work of [20]. Therefore, UKF is a method of estimation of the state of linear and nonlinear systems

Other studies show that more than one AUV can also be beneficial in navigating. As seen in the study [21]. A leader-follower formation of AUVs was used as a formation of these AUVs during underwater missions. This formation ensures that the follower stays on the path even without the leader's velocity. This works by applying Lyapunov with the backstepping synthesis in the tracking of the virtual leader formed by the followers based on the actual leader. With this, missions are much more coordinated and unknown 
disturbances on the follower are mitigated this also mitigates the trouble in communicating in underwater environments.

Lastly, the localization of an environment is also an integral part of the AUVs navigation system. To put it simply, localization is the familiarization of the AUV or other robotic equipment to the environment or place they are operating in. A study by [22] comprises of localization in man-made areas such as dams and harbors. The information obtained by the AUV's sensors runs through simultaneous localization and mapping (SLAM) using the EKF. With this, different experiments in the Costa Brava in Spain show the viability of such an approach. However, the study will not be limited to only man-made structures and further studies will be conducted on the feasibility in other marine environments.

\subsection{Imaging Capabilities of AUVs}

One of the key components in modern-day manned underwater vehicles is the sonar sensors [23]. This technology has also been applied to today's AUV. Their article talks about how to incorporate a better sonar system by incorporating the Simple Linear Iterative Clustering or SLIC in the imaging process to improve the accuracy of sonars. Then a homogeneity patch is applied from the homogenous super pixels from the SLIC. The researchers in the study then ran an adaptive intensity constraint through the Markov Random Field (MRF). Both of these are to segment the MRF at each iteration. This research proves to be effective in sonar images with intensity inhomogeneity. As it is pitch black in the lower parts of the ocean, better imagery is needed to better analyze different parts of the ocean floor without having to expose the AUV elements such as heat and chemicals found in extreme areas of the ocean bottom.

Other than existing sonar imagery for underwater vehicles, a recent study by [24] utilizes light stripe range (LSR) and photometric stereo (PS) to produce a high-density 3-Dimensional (3D) rendering of the AUV's surrounding. The system aims to reduce the disadvantages of having an independent photometric stereo. The output of the hybrid is an accurate 3D image of a close-range inspection of underwater scenarios. However, the system is only valid for close-range shots, with the sonar imaging on the other hand, able to predict 3D renders of the ocean in a much longer distance but suffers from quality loss. These imaging systems are what the AUV bases its operation on. One can state that these are the sensory organs of the AUV and what's responsible for certain actions of the autonomous vehicles.

\subsection{AUV Models}

A paper by [25] describes a docking and recharging station for the AUVs. This is because most of the AUVs used by scientists require constant refueling and data transfer manually through surfacing. Usually, these trips to manage AUVs can be quite costly considering the time and manpower required. The study introduces the "Marine Bird" AUV capable of docking and recharging by itself underwater. This method makes the AUV completely autonomous with human interference minimal and only limited to data transfer and maintenance. This study can also limit the frequency of communication with the AUV as instructions can be passed on to the docking station without having to directly communicate with the AUV. Not to mention that with today's technology, the AUV can be completely electrical and would lead to more environmentally friendly emissions.

In the topic of being environmentally friendly, a paper by [26] presents an AUV that is powered by the environment. Its thermal engine is powered by heat flow in the tropical environment it was tested on. However, this does not mean that the AUV can be used globally as there would not be enough heat on some parts of the globe. Another environmental energy is solar energy was considered by the study, but it was not as abundant and readily available as thermal energy.

The study of [27] outlines the development of a solar-powered AUV. It started back in 1998 where a solar-powered AUV the SAUV I was developed, it ran by using panels and NiCad batteries. In 2004 a newer SAUV II was developed due to advancements in solar technology. SAUV II was capable of long-term deployment and monitoring of the deep ocean. When charging its Lithium-ion batteries, the vehicle would simply float in the ocean surface. While floating the AUV would also be able to communicate with the people handling it. This eliminates the need for docking and recharging station as the AUV is self-sufficient enough.

However, an AUV designed to clean the ocean would not be applicable if it would have no means to collect marine debris. In the research conducted by [28], shows a device using a robotic arm to accomplish specified tasks in shallow water. However, underwater vehicles usually use an underwater manipulator. A study by [29] shows the difficulties in trying to obtain a fully autonomous underwater manipulator who intervenes in deep ocean operations. Tasks given to the manipulators were to recover an underwater object using a hook and to successfully bring the object to the surface.

\subsection{Controlling AUVs}

As the last piece of necessity when it comes to designing a proper autonomous vehicle, it is required that the vehicle must be capable of driving itself. As such, autopilot systems were developed. For an AUV, the autopilot system can also be referred to as the AUV's hydrodynamics. Some works outline these autopilot systems such as the study of [30]. Other studies show how different approaches or methods were used to produce the autopilot system, such as the ANFIS approach by [31], where he used neuro-fuzzy techniques. With the addition of tech from the modern world, studies have also been able to maximize the use of the autopilot to the precise stuff. A study by [32] shows the homing in and docking of the AUV using autopilot. A study by [33] even shows the use of artificial intelligence or AI in autopilots in ships. The AI is used in powerful simulations that aid the perfection of the ship's autopilot. 


\section{THEORETICAL CONSIDERATIONS}

Because of the tendency of the ocean to block nearly every form of communication between the AUV and the host. These robots must have the capability to adjust to certain situations. With that in mind, autopilots and machine learning are the best bets of AUVs against these situations. Especially in cleanups, debris or dirt can sometimes cause a malfunction of a system. It would be hard for the host to properly navigate the AUV because of the lack of communication tools available as of yet.

\section{DATA AND RESULTS}

With the review of literature in mind, we must note that the AUV will face multiple obstacles in properly operating. For one, underwater communication will be less prevalent meaning that once the AUV malfunctions during a mission, it will be a difficult task in retrieving it. With the AUV weakness out of the way, the researchers believe that the optimal AUV specifications for waste management will be that it supports solar power and applies the same recharging and data transferring of that of SAUV II. The AUV should be able to use the latest imaging system to properly scan for multiple floating debris or waste, such an imaging system can be the LSR with PS. This will aid the vehicle in finding wastes around the vehicle. Together with the SLIC processing of the AUV's sonar, information gathered can be used to localize the AUV in its stationed area. The AUV should be able to use underwater manipulators to collect large debris and to be able to store this in docking stations. Lastly, the AUV should have a proper autopilot system to efficiently maneuver and navigate in situations where no human interference is possible.

\section{ANALYSIS OF DATA}

As debris is scattered across the ocean, an AUV can likely be deployed to regions without proper infrastructure near the station. The lack of a proper station can mean that the AUV must be self-sufficient and would require little to no continuous human intervention. This is why it would be more preferred if the AUV would be a solar powered one since it would mean that refueling and docking can be done autonomously. AUVs that are to be used for ocean cleanups are to be resilient in many of the elements it will be exposed to. As such smart improvements in the navigation and imaging of the vehicle must be considered. Techniques such as the LSR and PS must be used to gain as much information as possible. This is for the machine to be able to use its SLIC to process the necessary information. The feedback gained from the SLIC tells the AUV the next step or action to be performed. Such actions can be the movement of the AUV's underwater manipulators in which the system detects that marine debris is spotted and is to be collected for proper disposal. However, before this activity can be processed, the machine has to have the proper autopilot to process the feedback from the SLIC. The autopilot is what translates to the machine the different steps to be undertaken. With the application of multiple AUVs in sync with each other, cleanups can be more efficient.

\section{CONCLUSION}

Due to the effects of water pollution on aquatic lifeforms, researchers have sought many answers to the problem at hand. $10 \%$ of such plastics end up in oceans waiting to be fed on by marine animals. The use of AUVs in cleaning the deep parts of the ocean is a step to a better future. With the ability to use multiple AUVs in cleaning the ocean, it can also be said that the process can be much faster due to available technologies. Moreover, the systems used in the past have been replaced by more efficient and precise systems. With these advancements, the use of AUVs in ocean cleanup is much more reliable.

To finally conclude, the use of AUVs in underwater manipulation and interventions comes with its own set of obstacles, however, with the application of new technologies such as the autopilot system or the new imaging techniques all help give the AUVs more accurate control. With the introduction of artificial intelligence can help aid the formation of smarter autopilot systems.

\section{RECOMMENDATIONS}

Through thorough research, various studies and experiments have given fruitful knowledge and contribution to creating efficient Autonomous Underwater Vehicles. The current studies, programs, and designs available of these Autonomous Underwater Vehicles have been of cutting-edge technology and up to date innovations; however, there are still many ways to improve on the technology. First, the group recommends that the body of the AUV should be made from recycled or environment-friendly material so long that it may still withstand the strong currents and obstacles of the ocean. Since one of the main goals of an AUV is to be able to monitor the state of the environment to help study and find solutions to the degrading ecosystem the device must be also made out of hazard-free materials. Second, the group recommends that further studies be done to prolong the energy and power capacity of an AUV. AUVs are known to consume a great amount of energy and knowing that some paths and obstacles in remote areas may be difficult to reach this may be an important parameter to maximize the efficiency and utility of the device. Third, although the model and system are efficient, through thorough research there still may be ways to improve the program by going through the important parameters that an AUV may encounter, this can be done by modeling the system to detect accurate, precise and clear data About this, further research on the needs of the environment must be considered to ensure that the data collected is coherent and is of use. Also, although many studies have provided the AUVs to be completely autonomous there are still countless loopholes that must be considered. Thus, the fact that unexpected discrepancies in the system should be considered, the researchers suggest that future researchers and engineers develop a model with an improved system such it is programmed to detect. Last but not least, AUVs can be used in so many fields to be able to curate and innovate a device, 
ensure that the study is focused on a certain goal and under certain parameters this then enables one to really determine the faults and develop the device to its best version.

\section{REFERENCES}

[1] C. HuetandF. Mastroddi, "Autonomy for underwater robots-a European perspective," Autonomous Robots. Vol. 40, No. 7, pp. 1113-1118, 2016. https://doi.org/10.1007/s10514-016-9605-x

[2] J. Gelli, A. Meschini, N. Monni, M. Pagliai, A. Ridolfi, L. Marini and B. Allotta, "Development and design of a compact autonomous underwater vehicle: Zeno AUV," IFAC-PapersOnLine. Vol. 51, No.29, pp. 20-25, 2018. https://doi.org/10.1016/j.ifacol.2018.09.463

[3] A. Carr, "Impact of nondegradable marine debris on the ecology and survival outlook of sea turtles," Marine Pollution Bulletin. Vol.18, No.6, pp. 352-356, 1987. https://doi.org/10.1016/S0025-326X(87)80025-5

[4] S. C. Gall and R. C. Thompson, "The impact of debris on marine life," Marine Pollution Bulletin. Vol.92, Nos. 1-2, pp. 170-179, 2015.

[5] C. M. Boerger, G. L. Lattin, S. L. Moore and C. J. Moore, "Plastic ingestion by planktivorous fishes in the north pacific central gyre." Marine Pollution Bulletin. Vol.60, No.12, 2010. https://doi.org/10.1016/j.marpolbul.2010.08.007

[6] H. S. Carson, S. L. Colbert, M. J. Kaylor and K. J. McDermid, "Small plastic debris changes water movement and heat transfer through beach sediments," Marine Pollution Bulletin. Vol.62, No.8, pp. 1708-1713, 2011.

[7] R. B. Wynn, V. A. I. Huvenne, T. P. Le Bas, B. J. Murton, D. P. Connelly, B. J. Bett and J. E. Hunt, "Autonomous underwater vehicles (AUVs): Their past, present and future contributions to the advancement of marine geoscience," Marine Geology. Vol.352, pp. 451-468, 2014.

https://doi.org/10.1016/j.margeo.2014.03.012

[8] I. Segovia, A. Pliego, M. Papaelias and F. P. García Márquez, "Optimal management of marine inspection with autonomous underwater vehicles," 2020.

[9] G. Xu, W. Shen and X. Wang, "Marine environment monitoring using wireless sensor networks: A systematic review," 2014.

[10]H. Xu, G. Zhang, Y. Sun, S. Pang, X. Ran and X. Wang, "Design and experiment of a plateau data-gathering AUV," Journal of Marine Science and Engineering. Vol.7, No.10, 2019. https://doi.org/10.3390/jmse7100376

[11] A. Cózar, F. Echevarría, J. I. González-Gordillo, X. Irigoien, B. Úbeda, S. Hernández-León and C. M. Duarte, "Plastic debris in the open ocean," Proceedings of the National Academy of Sciences of the United States of America. Vol.111, No.28, pp. 10239-10244, 2014.

[12] G. M. Quero and G. M. Luna, "Surfing and dining on the "plastisphere": Microbial life on plastic marine debris," Advances in Oceanography and Limnology. Vol.8, No.2, pp. 199-207, 2017.
[13] S. S. Myers, "Global environmental change: The threat to human health," Worldwatch Paper. Vol. 181, pp. 1-48, 2009.

[14] A. Sahoo, S. K. Dwivedy and P. S. Robi, "Dynamic Modelling and Control of a Compact Autonomous Underwater Vehicle," Lecture Notes in Mechanical Engineering. 2020. https://doi.org/10.1007/978-981-13-8323-6_25

[15] E. Gai, "The century of inertial navigation technology," IEEE Aerospace Conference Proceedings. Vol.1, pp. 59, 2000.

[16] H. He, J. Xu, F. Qin and F. Li, "Platform bench strap-down algorithm-inertial navigation system," Proceedings - 2015 Chinese Automation Congress. Pp. 1550-1554, 2016.

[17] A. Africa, F. Asuncion, J. Tiberio and R.Munchua,"Global positioning system using mobile phones: Three-dimensional positioning," International Journal of Emerging Trends in Engineering Research. Vol. 8, No. 2, pp. 295-299, 2020. https://doi.org/10.30534/ijeter/2020/09822020

[18] I. S. Al-Kofahi, Z. Albataineh, M. Tantawi and A. Dagamseh, "The narrowband tunable radio frequency (RF) power amplifier with high-efficiency at $2.4 \mathrm{GHz}$ frequency," International Journal of Emerging Trends in Engineering Research. Vol. 8, No. 3, pp. 763-768, 2020. https://doi.org/10.30534/ijeter/2020/25832020

[19] B. Allotta, A. Caiti, R. Costanzi, F. Fanelli, D. Fenucci, E. Meli and A. Ridolfi, "A new AUV navigation system exploiting unscented Kalman filter," Ocean Engineering. Vol. 113, pp. 121-132, 2016.

[20] S. J. Julier and J. K. Uhlmann, "New extension of the Kalman filter to nonlinear systems," Proceedings of SPIE - the International Society for Optical Engineering. No. 3068, pp. 182-193, 1997. https://doi.org/10.1117/12.280797

[21]R. Cui, S. S. Ge, B. Voon Ee How and Y. Sang Choo, "Leader-follower formation control of underactuated autonomous underwater vehicles," Ocean Engineering. Vol.37, Nos.17-18, pp. 1491-1502, 2010.

[22] H. Wang, J. Wang, L. Yu and Z. Liu, "A new SLAM method based on SVM-AEKF for AUV,"OCEANS'11 MTS/IEEE Kona. 2011.

[23] Y. Song and P. Liu, "Segmentation of sonar images with intensity inhomogeneity based on improved MRF," Applied Acoustics.Pp. 158, 2020.

[24] A. M. Pinto and A. C. Matos, "MARESye: A hybrid imaging system for underwater robotic applications," Information Fusion. Vol.55, pp. 16-29, 2020. https://doi.org/10.1016/j.inffus.2019.07.014

[25] T. Fukasawa, T. Noguchi, T. Kawasaki and M. Baino, "Marine bird: A new experimental AUV with underwater docking and recharging system," Oceans Conference Record (IEEE). No.4, pp. 2195-2200, 2003.

[26] D.C. Webb, P. J.Simonetti and C. P. Jones, "SLOCUM: An underwater glider propelled by environmental energy," IEEE Journal of Oceanic Engineering.Vol. 26, No. 4, pp. 447-452, 2001. https://doi.org/10.1109/48.972077 
[27] D. Blidberg, S. Mupparapu, S. Chappell, R. Komerska, J. C. Jalber and R. Nitzel, "The SAUV II (solar-powered AUV) test results 2004," Oceans 2005 - Europe. No. 1, pp. 545-550, 2005.

[28] J. J. Fernández, M. Prats, P. J. Sanz, J. C. García, R. Marín, M. Robinson and P. Ridao, "Grasping for the seabed: Developing a new underwater robot arm for shallow-water intervention," IEEE Robotics and Automation Magazine. Vol. 20, No. 4, pp. 121-130, 2013. https://doi.org/10.1109/MRA.2013.2248307

[29] G. Marani, S. K. Choi and J. Yuh, "Underwater autonomous manipulation for intervention missions AUVs," Ocean Engineering. Vol. 36, No. 1, pp. 15-23, 2019.

[30]M.L. Graves II and D. E. Humphreys, "Hydrodynamic/autopilot design of an AUV," Proceedings of MTS/IEEE OCEANS. 2005.

[31]R. Sutton and P. J. Craven, "The ANFIS approach applied to AUV autopilot design," Neural Computing and Applications. Vol. 7, No. 2, pp. 131-140, 1998. https://doi.org/10.1007/BF01414165

[32] V. K. Pshikhopov, M. Y. Medvedev and B. V. Gurenko, "Homing and docking autopilot design for autonomous underwater vehicle," 2014.

[33] W. Rekdalsbakken and A. Styve, "Simulation of intelligent ship autopilots," Proceedings - 22nd European Conference on Modelling and Simulation. Pp. 188-194, 2008.

https://doi.org/10.7148/2008-0188 